\section{(A) OPEN ACCESS}

\title{
Multimodal endovascular management of acute ischemic stroke in patients over 75 years old is safe and effective
}

\author{
George M Ghobrial, Nohra Chalouhi, Lana Rivers, Samantha Witte, Justin Davanzo, \\ Richard Dalyai, Michelle L Gardecki, Pascal Jabbour, Fernando Gonzalez, \\ Aaron S Dumont, Robert H Rosenwasser, Stavropoula Tjoumakaris
}

Department of Neurological Surgery, Thomas Jefferson University Hospital, Philadelphia, Pennsylvania, USA

\section{Correspondence to}

Dr S Tjoumakaris, Department of Neurological Surgery, Thomas Jefferson University, 909 Walnut Street, 2nd Floor, Philadelphia, PA 19107, USA: stavropoula.tjoumakaris@ jefferson.edu

Received 16 May 2012 Revised 14 June 2012 Accepted 16 June 2012 Published Online First 11 July 2012

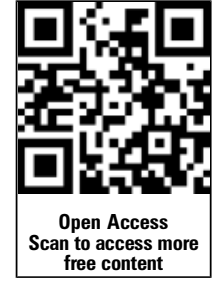

\begin{abstract}
Introduction Greater attention has been directed to endovascular recanalization of acute ischemic stroke in septuagenarians and above.

Technique A retrospective chart review was conducted
\end{abstract} to include patients treated for acute ischemic stroke from 2006 to 2012. All patients underwent initial neurological assessment and non-contrast head CT. Patients treated from 2009 to 2012 additionally received emergent CT angiogram and CT perfusion. 51 patients met the clinical and radiographic criteria and underwent multimodal endovascular revascularization for acute ischemic events. Results All patients underwent cerebral angiography and met angiographic criteria for endovascular thrombolysis. 34 patients $(67 \%)$ were older than 80 years of age. 23 patients $(45 \%)$ received intravenous tissue plasminogen activator prior to admission. Eight (16\%) patients underwent stent placement after intra-arterial thrombolysis, 10 (20\%) underwent balloon angioplasty and seven (14\%) underwent both angioplasty and stent placement. $21(41 \%)$ required only intra-arterial thrombolytics. An improvement in Thrombolysis in Myocardial Infarction score was noted in 34 patients (67\%). The average modified Rankin Scale score on discharge was 3.9. Symptomatic intracranial hemorrhage occurred in three patients (6\%); none required surgery. One patient (1.9\%) had a postoperative retroperitoneal hematoma, which was managed conservatively. Two fatalities resulted from intraoperative vessel rupture (3.9\%), with a combined morbidity and mortality of $27.5 \%$. Conclusions Multimodal endovascular recanalization of acute ischemic stroke is a relatively safe treatment option in patients older than 75 years of age. Careful patient selection by clinical and radiographic inclusion criteria is necessary for the successful management of stroke in this age group.

\section{INTRODUCTION}

Stroke is the third leading cause of death in the USA, and its prevalence is increasing. ${ }^{1-4}$ Our demographic findings include an increase in the elderly age category of greater than 65 years of age, with a corresponding increase in the prevalence of stroke in the elderly. ${ }^{4}$ With advances in intra-arterial and mechanical thrombolysis, the interventional window was first extended to $8 \mathrm{~h}$. Recently, the use of CT perfusion (CTP) has supported multimodal recanalization beyond $8 \mathrm{~h}$. As a result, more elderly patients are participating in endovascular treatment of acute ischemic stroke. ${ }^{5}$ CTP supports interventions in late diagnosis and distant referrals from stroke centers lacking in neurointerventional resources. ${ }^{6}$

Intuitively, minimally invasive treatment would seem to be better tolerated in the elderly population, but data have not supported this hypothesis to date. Also, endovascular intervention in the elderly suffering from acute ischemic stroke has not been thoroughly investigated. Kim et $a l^{7}$ in a retrospective study comparing 31 elderly patients undergoing intra-arterial thrombolysis with a cohort of less than 80 years old, found no significant differences in recanalization or intracerebral hemorrhage, although a lower rate of survival and excellent outcome was noted in the elderly group. Similarly, Loh et $a l^{8}$ found a higher likelihood of death among the elderly cohort after recanalization, with similar rates of recanalization and intracranial hemorrhage. Quereshi et $a l^{9}$ in a pooled analysis of four prospective studies of thrombolysis in the elderly found in 24 patients a lower likelihood of recanalization and favorable outcome (modified Rankin Scale (mRS) score 0-2) along with increased mortality rates.

Elderly patient populations need to be studied with regard to interventional stroke care to determine if traditional therapies can be carried out without added risk. Angioplasty and stenting are increasingly performed alongside mechanical and chemical thrombolysis to maximize recanalization and improve flow restoration, with favorable results ${ }^{10-12}$ Previous studies were limited by their small sample size. We assessed the safety and efficacy of multimodal endovascular recanalization in series of 51 patients aged $\geq 75$ years who were treated in our institution.

\section{METHODS}

Institutional review board approval was obtained. A retrospective chart review was conducted to include patients over the age of 75 years who were treated for acute ischemic stroke in our institution during the period 2009-2012. All patients underwent initial neurological assessment. Preoperative evaluation included a non-contrast head CT, which provided a baseline and excluded patients with intracranial hemorrhage or completed infarctions. 
In the period between 2006 and 2012, all patients underwent additional emergent CT angiography and CTP studies. The decision to proceed with endovascular thrombolysis was determined based on clinical and radiographic analysis of preoperative data. Inclusion criteria for intervention were a minimum National Institutes of Health Stroke Scale score of 8, CTP with ischemic penumbra on mean transient time and cerebral blood flow but preservation of cerebral blood volume, and detectable arterial occlusion on CT angiography. All arterial occlusions were confirmed by subsequent digital subtraction angiography. CTP has seen increased popularity for rapid assessment of candidates for endovascular intervention although its routine use in clinical practice is still under debate. ${ }^{13-25}$

Analysis of patient data included inpatient hospital records, imaging studies, operative reports and outpatient office records. Patient demographics, admission and discharge neurological examinations, medical comorbidities, such as prior history of hypertension, diabetes, stroke and smoking, and treatment complications were recorded and analyzed. Clinical outcome was evaluated at time of discharge using the mRS.

A total of 51 patients met the clinical and radiographic criteria and underwent multimodal endovascular recanalization for acute ischemic events. Postoperative course was assessed by MRI evaluation of stroke territory, incidence of intracranial hemorrhage and clinical neurological outcome on discharge. All CT and MRI data were evaluated by staff neuroradiologists. Thrombolysis in Myocardial Infarction (TIMI) scores were classified as follows: TIMI 0, no flow beyond the thrombus; TIMI 1 , penetrance beyond the thrombus but without perfusion; TIMI 2, delayed perfusion; TIMI 3, normal perfusion beyond the thrombus. Time to intervention was defined as time from symptom onset to groin puncture.

Data acquired were analyzed by the Mann-Whitney $U$ test and $\chi^{2}$ analysis where appropriate, using a statistical software package (MedCalc V.12.2.1.0 Mariakerke, Belgium).

\section{Endovascular treatment}

Endovascular procedures occurred under general endotracheal anesthesia. Digital subtraction angiography of the affected carotid circulation was performed. An 8 French sheath and an 8 French guide catheter were used in most cases. An SL-10 (Stryker, Fremont, California, USA) and Synchro-14 microguidewire (Stryker) were used to cross the occluded arterial segment and determine the extent of the thrombus. Proximal internal carotid artery (ICA) occlusions were revascularized with balloon angioplasty with Viatrac (Abbott, Santa Clara, California, USA) and Aviator (Cordis, Bridgewater, New Jersey, USA) balloons. Off-label use of proximal carotid stenting was achieved with Precise (Cordis), Acculink (Abbott) and Guidant (Boston Scientific, Natick, Massachusetts, USA) stents. Once the cervical carotid circulation was re-established, angiography was repeated to assess the intracerebral circulation. If a tandem occlusion was seen in the intracranial circulation, mechanical thrombolysis was undertaken via a Merci Retriever (Concentric Medical, Mountainview, California, USA), Penumbra System (Penumbra Inc, Alameda, California, USA) $(\mathrm{n}=3)$, Hyperglide balloon (EV3, Irvine, California, USA) and Gateway balloon (Stryker). If mechanical thrombolysis was unsuccessful and ictus was within $8 \mathrm{~h}$, pharmacologic thrombolysis was performed with intra-arterial tissue plasminogen activator, urokinase or ReoPro. The use of arterial thrombolytics has shifted throughout the time period reported. Urokinase was predominantly utilized in 2006 but has seen a steady decline in its use in favor of tissue plasminogen activator. For thrombi that are resistant to lytics, ReoPro was sometimes used at the discretion of the interventionalist. Additional intracranial stenting for tandem middle cerebral artery occlusions was performed with Wingspan (Stryker) and Enterprise (Cordis) stenting. The offlabel use of the Enterprise stent has gained popularity for stroke intervention, due to its improved navigability and ease of deployment in various vessel anatomy (often not navigable with the Wingspan). ${ }^{26}$ Finally, in all patients, post thrombolysis cerebral angiography was repeated to assess the patency of the extracranial and intracranial carotid circulation.

\section{Postoperative management}

Postoperatively, all patients were transferred to the neurointensive care unit for a minimum of $24 \mathrm{~h}$, after which a noncontrast head CT was routinely performed. All patients who underwent stent assisted revascularization and had no contraindication to antiplatelet therapy received $75 \mathrm{mg}$ of clopidogrel daily for 6 weeks, as well as $81 \mathrm{mg}$ of aspirin daily, which was continued indefinitely.

\section{RESULTS}

All patients underwent cerebral angiography and met the angiographic criteria for endovascular thrombolysis. There were 35 women and 16 men with a mean age of 81.4 years (range $75-90$ years). Thirty-four patients $(67 \%)$ were $>80$ years of age. Thirty-one $(60.8 \%)$ patients had arterial hypertension, 23 (45.1\%) had hypercholesterolemia, 12 (23.5\%) had diabetes mellitus, $23(45.1 \%)$ had atrial fibrillation and seven (13.7\%) were active smokers. Mean National Institutes of Health Stroke Scale score was 17.1. The middle cerebral artery was occluded in 39 (76.5\%) patients, the ICA in $10(19.6 \%)$ patients and the basilar artery in two (3.9\%) patients. Twenty-three patients $(45 \%)$ received intravenous tissue plasminogen activator prior to admission. Mean time from symptom onset to endovascular intervention was $6.1 \mathrm{~h}$. In 41 patients $(80.4 \%)$, more than one endovascular treatment modality was employed (tables 1 and 2). The penumbra device was used in 23 patients, the Merci device in nine and both devices in 13 . Eight $(16 \%)$ patients underwent stent placement, $10(20 \%)$ underwent balloon angioplasty and seven $(14 \%)$ underwent both angioplasty and stent placement. Twenty-one $(41 \%)$ patients required intra-arterial thrombolytics. At baseline, $46(90.2 \%)$ patients had a TIMI score of 0 or 1 (mean TIMI score was 0.19 ). An improvement in TIMI score was noted in 34 patients (67\%). In 51 patients, postrecanalization TIMI scores were 0 in 11 (21.5\%) patients, 1 in seven (13.7\%) patients, 2 in $14(27.5 \%)$ patients and 3 in 19 (37.3\%) patients. A total of $13(25.4 \%)$ patients demonstrated some degree of intracranial hemorrhage on postoperative imaging. Nine of 13 hemorrhages were confluent hematomas (PH-1 and PH-2, by the ECASS (European Cooperative Acute Stroke Study) classification). ${ }^{27}$ Symptomatic intracranial hemorrhage occurred in three patients $(6 \%)$ but did not require surgical intervention. One patient $(1.9 \%)$ had a postoperative retroperitoneal hematoma, which was managed conservatively. There were two fatalities in our series from intraoperative vessel rupture (3.9\%). The average length of stay was 11.8 days (1-32). At time of discharge, $33.3 \%$ of patients had a favorable outcome (mRS score $0-3$ ). The average $\mathrm{mRS}$ score was 3.9. The all cause death rate was $21.6 \%$ in the series.

\section{DISCUSSION}

Acute ischemic stroke is the third leading cause of death in the USA and the most prevalent neurologic diagnosis. It is also the most common diagnosis in nursing home residents. With 
Table 1 Patient characteristics

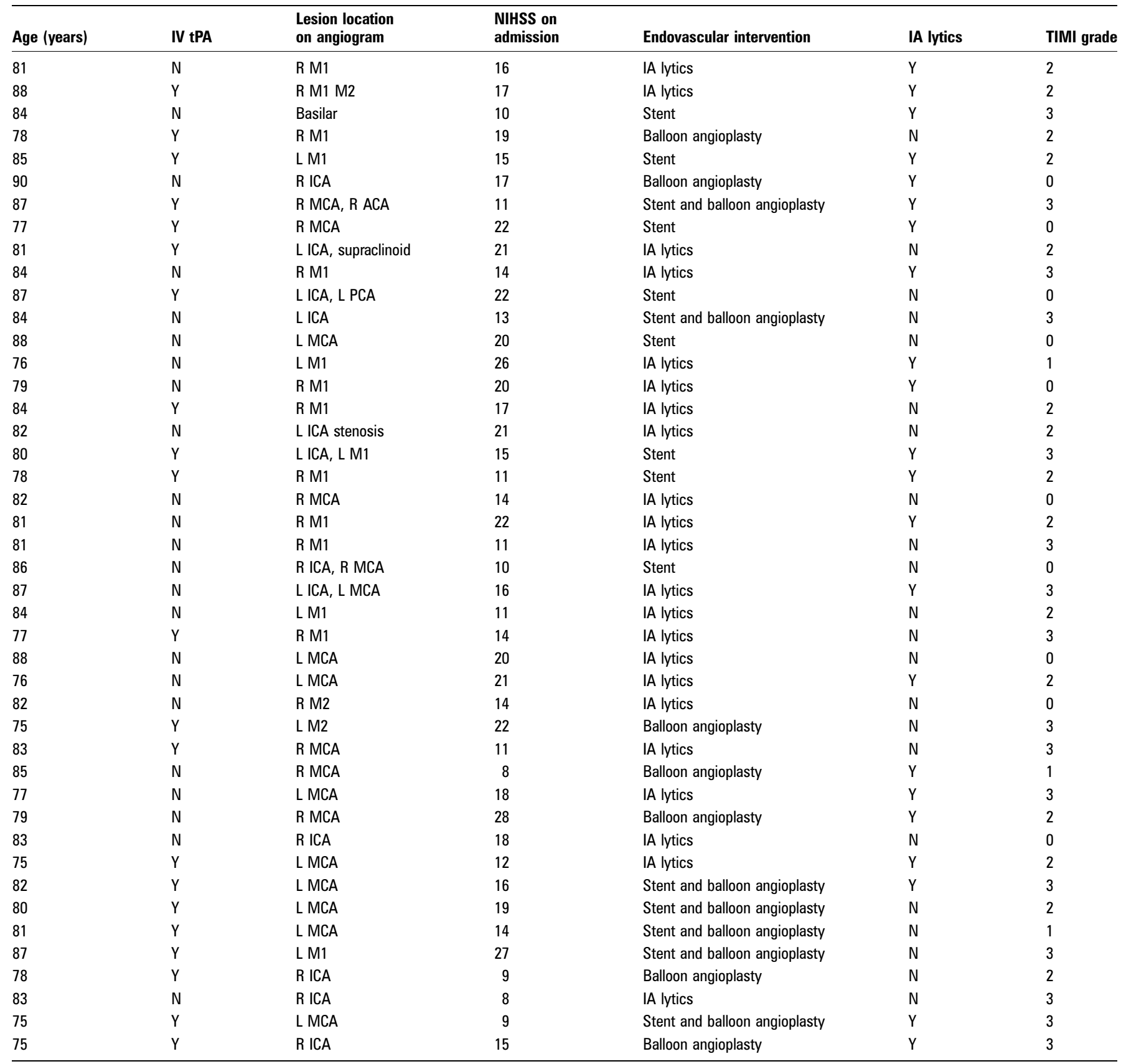

ACA, anterior cerebral artery; IA, intra-arterial; ICA, internal carotid artery; IV tPA, intravenous tissue plasminogen activator; L, left; MCA, middle cerebral artery; NIHSS, National Institutes of Health Stroke Scale; PCA, posterior cerebral artery; R, right; TIMI, Thrombolysis in Myocardial Infarction.

advancements in medicine and prolongation of life expectancy, stroke commonly affects the elderly. Unfortunately, there are minimal data to support acute endovascular intervention in this patient population. Additional studies are currently underway with the recent publication of the Thrombolysis in Elderly Stroke Patients in Italy (TESPI), a randomized, controlled trial evaluating intravenous tissue plasminogen activator versus standardized medical therapy in patients $>80$ years of age, which is currently underway. ${ }^{28}$

Existing medical comorbidities such as diabetes, hypertension, cardiac history and renal insufficiency increase perioperative patient risk. Furthermore, vascular anatomical limitations, such as diffuse atherosclerosis, vessel tortuosity and peripheral vascular disease, pose additional surgical risks in the elderly. Yet, rapid reperfusion of the cerebral penumbra remains the gold standard in acute stroke care. The Carotid Revascularization Endarterectomy versus Stenting Trial (CREST) supported the notion that ICA stenting is comparable with carotid endarterecomy in terms of perioperative morbidity and mortality after 30 day and 2.5 year follow-ups. ${ }^{28}$ Later analysis of the CREST data suggested a correlation between increasing age and risk of stroke. ${ }^{28} 29$

In our series, we investigated the safety and efficacy of multimodality endovascular intervention for acute ischemic stroke in septuagenarians and above. Due to the high surgical risk in this patient population, careful inclusion criteria were set. The angiographic revascularization rates were good, with a mean TIMI score of 2 post-recanalization, with 34 patients (67\%) achieving TIMI scores of 2 or greater. Perioperative morbidity was $27.5 \%$, due to 13 intracranial and one retroperitoneal hemorrhage. Only three $(5.8 \%)$ of the 13 intracranial hemorrhages were symptomatic. This was less than half the reported rate of symptomatic intracranial hemorrhage in an analysis of the PROACT II 
Table 2 Baseline characteristics

\begin{tabular}{llccl}
\hline & Total & Age $<\mathbf{8 0}$ years & Age $>\mathbf{8 0}$ years & p Value \\
\hline Male $(\mathrm{n}(\%))$ & 14 & 6 & 8 & 0.95 \\
Female $(\mathrm{n}(\%))$ & 37 & 11 & 26 & 0.06 \\
Atrial fibrillation & 23 & 4 & 19 & 0.04 \\
Diabetes & 12 & 3 & 9 & 0.38 \\
Hypertension & 31 & 23 & 8 & 0.04 \\
IV tPA & 23 & 10 & 13 & 0.8 \\
NIHSS & & 17.8 & 15.7 & 0.81 \\
Time intervention (h) & & 6 & 6.8 & 0.18 \\
\hline
\end{tabular}

NIHSS, National Institute of Health Stroke Scale; IV tPA, intravenous tissue plasminogen activator

(Prolyse in Acute Cerebral Thromboembolism II) study. ${ }^{30}$ In the aforementioned study, blood glucose $>200 \mathrm{mg} / \mathrm{dl}$ was associated with a $36 \%$ increased risk of intracranial hemorrhage. The prevalence of diabetes type I or II was higher in our population $(23.5 \%)$, but in our case, this did not increase the rate of hemorrhage. The addition of angioplasty and or stent placement did not appear to have an impact on the overall incidence of intracranial hemorrhage, despite the need for clopidogrel postintervention. Thirty-four per cent of patients underwent stent placement. While off-label, stent placement has increasingly been performed in acute stroke, to maximize flow restoration, with favorable results. ${ }^{10-12}{ }^{31-33}$ In the non-operative population, hemorrhagic conversion has been reported to range from $15 \%$ to as high as $43 \%{ }^{34}$ Our procedural death rate was $3.9 \%$, which was due to vessel rupture, as evidenced by contrast extravasation intraoperatively. In previous series of elderly patients, time from ictus to endovascular intervention ranged from a mean of 4.3 to $6.25 \mathrm{~h}^{21}{ }^{35}$ Our study had an average time to intervention of $6.1 \mathrm{~h}$. As a tertiary referral center, most patients at our center are evaluated at an outside institution and then transferred on clinical suspicion of stroke. The use of CTP criteria (which allowed the extension of the therapeutic window) to select patients for endovascular intervention also explains the long interval from stroke onset in our study.

Recently, Galimanis et al prospectively followed 623 patients who underwent endovascular recanalization for stroke. They concluded that 'time to treat' was the only significant factor leading to a better outcome. ${ }^{36}$ Of one of the largest series to date involving multimodal recanalization, they found a $5.5 \%$ incidence of symptomatic intracranial hemorrhage. Qureshi et al in his study of patients over 80 years old found a rate of $12 \%$ intracranial hemorrhage. We found 13 hemorrhages $(25.4 \%)$ on postoperative routine imaging. Almost half of these patients had stent placement. A similarly high rate of hemorrhage was also reported by Loh et $\mathrm{al}^{8}(61 \%)$. Difficulty arises in comparing rates of hemorrhage after intervention for a number of reasons. In our series, the added risk of hemorrhage from stent placement is unknown. Most importantly, $45 \%$ of our elderly cohort received intravenous tissue plasminogen activator prior to treatment, compared with $13 \%$ in the series by Qureshi and 35\% by Loh et $a l^{8}$ (intra-arterial and intravenous combined). Use of intra-arterial lytics to date has not used a standardized dose, which also does not allow for an even comparison across these three studies. Lastly, Loh et al utilized thrombectomy in all of their patients, without the use of stent. The observed morbidity and mortality in our study (21\%) compares favorably with those of Qureshi et al (54\%) and Loh et al ${ }^{8}$ (48\%).

The goal of stent placement in the setting of acute ischemic stroke is recanalization of the occluded vessel. Recanalization has been shown by Rha et $a l^{37}$ to have a favorable clinical impact on meta-analysis. A retrospective review of 115 patients by Al-Ali et al showed that angioplasty had a lower 1 year risk of recurrent or new stroke than medical management with warfarin and aspirin. In their study, the risk of stroke was very similar when comparing bare metal stent to medical management (19\% vs $18 \%$, respectively). ${ }^{38}$ Stenting for flow restoration in stroke is feasible with high rates of recanalization. ${ }^{5}$ Stampfl et al showed successful recanalization with stenting in $89 \%$ in acute stroke. ${ }^{5}$ Brekenfield et al followed prospectively 40 consecutive patients with acute ischemic stroke; $43 \%$ of patients underwent Wingspan (Boston Scientific) stenting and $57 \%$ of patients underwent thrombectomy alone with the Solitaire FR revascularization device (ev3, Plymouth, Minnesota, USA). Median ages were comparable (66 and 68 years in the two groups, respectively). The stented group showed superior rates of recanalization, and earlier time to flow restoration is seen, which were statistically significant. ${ }^{39}$ Compared with thrombolysis alone, stent placement demonstrated higher rates of recanalization as well as comparable morbidity. ${ }^{40}$

The median age in this group was 81 years. One would expect a higher morbidity when the results of stroke studies are translated clinically. Stenting requires a minimum vessel compliance to achieve vessel recanalization safely. In human studies, Van Lammeren et al ${ }^{41}$ harvested 1385 carotid plaques following endarterectomies, finding a correlation between larger more calcified plaques among the elderly. They showed that plaque stability decreased with age. Consequently, the endovascular risk during mechanical thrombolysis in the elderly may be higher than in the general population. In our patient group, two patients died as a result of intraoperative rupture following balloon angioplasty of the distal cervical ICA. Despite the advanced age in our series, our combined morbidity and mortality of $27.5 \%$ in the practice of multimodal recanalization did not differ significantly from that reported in younger patients. ${ }^{9}$ Mathews et $a l^{42}$ reviewed 94 consecutive patients who underwent endovascular therapy after ischemic stroke and found a combined morbidity and mortality of $26.6 \%$. Again, given the lack of standardization in new interventional techniques, comparing results across studies poses a challenge.

On discharge, 17 patients (33\%) had an mRS score of equal or less than 3, compared with $29 \%$ and $16 \%$ found by Loh ${ }^{18}$ and Qureshi ${ }^{20}$ on discharge, respectively. While the mean age of the patient population was $>80$ years, 17 patients were between the ages of 75 and 80 years. As our series had equivalent to better outcomes than those previously reported, this potential bias should be noted. In fact, we felt that choosing an arbitrary cut-off of 75 as opposed to 80 years would provide a larger patient population for study. The number of patients older than 80 years $(n=34)$ still comprises $67 \%$ of our patient population and therefore the results should be easily compared with existing studies.

General anesthesia during acute stroke intervention is standard practice at our institution at present. Recent literature raises the concern that general anesthesia may worsen neurologic outcomes, or even increase morbidity and mortality, compared with conscious sedation for endovascular recanalization. ${ }^{43}{ }^{44}$ However, these observations were reported by retrospective studies and thus require confirmation by large prospective randomized studies. Our study has some limitation pertaining to its retrospective design and the absence of a control group (younger patients). We recognize that given the increased risks associated with endovascular treatment in this age group and the strict inclusion criteria for intervention, the patient population is rather small. Assessing outcome at discharge may also 
not reflect ultimate patient outcome. As mentioned previously, given the single institutional nature of this study, results are interpretable mainly to specific protocols utilized at this institution. Use of angioplasty and stenting must also be considered when interpreting results. Larger prospective randomized studies are required to investigate acute stroke interventions in this patient population, as little exists in the literature.

\section{CONCLUSION}

Multimodal endovascular recanalization of acute ischemic stroke is a safe treatment option in patients $>75$ years of age. Careful patient selection by clinical and radiographic criteria is necessary for the successful management of stroke in this age group.

Contributors GMG, NC and RD were involved in manuscript writing, data collection and analysis. LR, SW, JD and MLG were involved in data collection and analysis. PJ, FG, ASD, RHR and ST were involved in manuscript preparation, data analysis, collection and interpretation.

Competing interests None.

Ethics approval The study was approved by the institutional review board.

Provenance and peer review Not commissioned; externally peer reviewed.

Open Access This is an Open Access article distributed in accordance with the Creative Commons Attribution Non Commercial (CC BY-NC 3.0) license, which permits others to distribute, remix, adapt, build upon this work non-commercially, and license their derivative works on different terms, provided the original work is properly cited and the use is non-commercial. See: http://creativecommons.org/licenses/by-nc/3.0/

\section{REFERENCES}

1. Bejot $\mathbf{Y}$, Touze E, Jacquin A, et al. Epidemiology of stroke. Med Sci (Paris) 2009;25:727-32.

2. Leoo T, Lindgren A, Petersson J, et al. Risk factors and treatment at recurrent stroke onset: results from the recurrent stroke quality and epidemiology (resque) study. Cerebrovasc Dis 2008;25:254-60.

3. Fisher M. Stroke and TIA: epidemiology, risk factors, and the need for early intervention. Am J Manag Care 2008;14:S204-11.

4. Bejot Y, Rouaud 0, Gentil A, et al. Stroke in elderly: what have we learned from stroke epidemiology in younger people. Rev Neurol (Paris) 2008;164:809-14.

5. Stampfl S, Hartmann M, Ringleb PA, et al. Stent placement for flow restoration in acute ischemic stroke: a single-center experience with the solitaire stent system. AJNR Am J Neuroradiol 2011;32:1245-8.

6. Perez de la Ossa-Herrero N. Early access to stroke referral centres offers clinical benefits: the stroke code. Rev Neurol 2008;47:427-33.

7. Kim D, Ford GA, Kidwell CS, et al. Intra-arterial thrombolysis for acute stroke in patients 80 and older: a comparison of results in patients younger than 80 years. AJNR Am J Neuroradiol 2007;28:159-63.

8. Loh Y, Kim D, Shi ZS, et al. Higher rates of mortality but not morbidity follow intracranial mechanical thrombectomy in the elderly. AJNR Am J Neuroradiol 2010;31:1181-5

9. Qureshi Al, Suri MF, Georgiadis AL, et al. Intra-arterial recanalization techniques for patients 80 years or older with acute ischemic stroke: pooled analysis from 4 prospective studies. AJNR Am J Neuroradiol 2009;30:1184-9.

10. Kim SM, Lee DH, Kwon SU, et al. Treatment of acute ischemic stroke: feasibility of primary or secondary use of a self-expanding stent (neuroform) during local intra-arterial thrombolysis. Neuroradiology 2012;54:35-41.

11. Guimaraens-Martinez L, Vivas-Diaz E, Sola-Martinez T, et al. Arterial recanalisation in acute stroke by means of a self-expanding stent. Rev Neurol 2009;48:555-6.

12. Brekenfeld C, Schroth G, Mattle HP, et al. Stent placement in acute cerebral artery occlusion: use of a self-expandable intracranial stent for acute stroke treatment. Stroke 2009:40:847-52.

13. Turk A, Magarik JA, Chaudry I, et al. CT perfusion-guided patient selection for endovascular treatment of acute ischemic stroke is safe and effective. J Neurointerv Surg 2012;4:261-5.

14. Johnson MM. Stroke and CT perfusion. Radiol Technol 2012;83:467CT-86CT.

15. Fox AJ, Symons SP, Howard P, et al. Acute stroke imaging: CT with CT angiography and CT perfusion before management decisions. AJNR Am J Neuroradiol 2012;33:792-4.

16. Fahmi F, Marquering HA, Streekstra GJ, et al. Differences in CT perfusion summary maps for patients with acute ischemic stroke generated by 2 software packages. AJNR Am J Neuroradiol Published Online First 3 May 2012. doi: 10.3174/ajnr. A3110.
17. Allmendinger AM, Tang ER, Lui YW, et al. Imaging of stroke: part 1, perfusion CT - overview of imaging technique, interpretation pearls, and common pitfalls. AJR Am J Roentgenol 2012;198:52-62.

18. Konstas AA, Wintermark M, Lev MH. CT perfusion imaging in acute stroke. Neuroimaging Clin N Am 2011;21:215-38, ix.

19. Kan PT, Snyder KV, Yashar P, et al. Utility of CT perfusion scanning in patient selection for acute stroke intervention: experience at university at buffalo neurosurgery-millard fillmore gates circle hospital. Neurosurg Focus 2011;30:E4.

20. Brant-Zawadzki MN, Brown DM, Whitaker LA, et al. Emerging impact of CTA perfusion CT on acute stroke thrombolysis in a community hospital. J Neurointervent Surg 2009;1:159-64.

21. Silvennoinen HM, Hamberg LM, Lindsberg PJ, et al. CT perfusion identifies increased salvage of tissue in patients receiving intravenous recombinant tissue plasminogen activator within $3 \mathrm{~h}$ of stroke onset. AJNR Am J Neuroradiol 2008;29:1118-23.

22. Rai AT, Carpenter JS, Peykanu JA, et al. The role of CT perfusion imaging in acute stroke diagnosis: a large single-center experience. J Emerg Med 2008;35:287-92.

23. Wintermark M, Flanders AE, Velthuis $B$, et al. Perfusion-CT assessment of infarct core and penumbra: receiver operating characteristic curve analysis in 130 patients suspected of acute hemispheric stroke. Stroke 2006;37:979-85

24. Wintermark M, Fischbein NJ, Smith WS, et al. Accuracy of dynamic perfusion CT with deconvolution in detecting acute hemispheric stroke. AJNR Am J Neuroradiol 2005;26:104-12.

25. Wintermark M. Brain perfusion-CT in acute stroke patients. Eur Radiol 2005;15 (Suppl 4):D28-31.

26. Chiam PT, Samuelson RM, Mocco J, et al. Navigability trumps all: stenting of acute middle cerebral artery occlusions with a new self-expandable stent. AJNR Am J Neuroradiol 2008;29:1956-8.

27. Renou $\mathbf{P}$, Sibon I, Tourdias $\mathrm{T}$, et al. Reliability of the ecass radiological classification of postthrombolysis brain haemorrhage: a comparison of CT and three MRI sequences. Cerebrovasc Dis 2010;29:597-604.

28. Hobson RW II, Howard VJ, Roubin GS, et al. Carotid artery stenting is associated with increased complications in octogenarians: 30 -day stroke and death rates in the crest lead-in phase. J Vasc Surg 2004;40:1106-11.

29. Mantese VA, Timaran $\mathrm{CH}$, Chiu D, et al. The Carotid Revascularization Endarterectomy versus Stenting Trial (CREST): stenting versus carotid endarterectomy for carotid disease. Stroke 2010;41:S31-4.

30. Kase CS, Furlan AJ, Wechsler LR, et al. Cerebral hemorrhage after intra-arterial thrombolysis for ischemic stroke: the proact ii trial. Neurology 2001;57:1603-10.

31. Levy El, Rahman M, Khalessi AA, et al. Midterm clinical and angiographic follow-up for the first food and drug administration-approved prospective, single-arm trial of primary stenting for stroke: saris (stent-assisted recanalization for acute ischemic stroke). Neurosurgery 2011;69:915-20.

32. Gupta R, Tayal AH, Levy El, et al. Intra-arterial thrombolysis or stent placement during endovascular treatment for acute ischemic stroke leads to the highest recanalization rate: results of a multicenter retrospective study. Neurosurgery 2011;68:1618-22.

33. Zaidat 00, Wolfe T, Hussain $\mathrm{SI}$, et al. Interventional acute ischemic stroke therapy with intracranial self-expanding stent. Stroke 2008;39:2392-5

34. Lyden PD, Zivin JA. Hemorrhagic transformation after cerebral ischemia: mechanisms and incidence. Cerebrovasc Brain Metab Rev 1993;5:1-16.

35. Konstas AA, Lev MH. Ct perfusion imaging of acute stroke: the need for arrival time, delay insensitive, and standardized postprocessing algorithms? Radiology 2010;254:22-5.

36. Galimanis A, Jung S, Mono ML, et al. Endovascular therapy of 623 patients with anterior circulation stroke. Stroke 2012;43:1052-7.

37. Rha JH, Saver JL. The impact of recanalization on ischemic stroke outcome: a meta-analysis. Stroke 2007;38:967-73.

38. Al-Ali F, Cree T, Duan L, et al. How effective is endovascular intracranial revascularization in stroke prevention? Results from borgess medical center intracranial revascularization registry. AJNR Am J Neuroradiol 32:1227-31.

39. Brekenfeld C, Schroth G, Mordasini P, et al. Impact of retrievable stents on acute ischemic stroke treatment. AJNR Am J Neuroradiol 32:1269-73.

40. Jovin TG, Gupta R, Uchino K, et al. Emergent stenting of extracranial internal carotid artery occlusion in acute stroke has a high revascularization rate. Stroke 2005;36:2426-30.

41. van Lammeren GW, Reichmann BL, Moll FL, et al. Atherosclerotic plaque vulnerability as an explanation for the increased risk of stroke in elderly undergoing carotid artery stenting. Stroke 2011;42:2550-5.

42. Mathews MS, Sharma J, Snyder KV, et al. Safety, effectiveness, and practicality of endovascular therapy within the first $3 \mathrm{~h}$ of acute ischemic stroke onset. Neurosurgery 2009;65:860-5.

43. Abou-Chebl A, Lin R, Hussain MS, et al. Conscious sedation versus general anesthesia during endovascular therapy for acute anterior circulation stroke: preliminary results from a retrospective, multicenter study. Stroke 2010;41:1175-9.

44. Davis MJ, Menon BK, Baghirzada LB, et al. Anesthetic management and outcome in patients during endovascular therapy for acute stroke. Anesthesiology 2012:116:396-405 